\title{
Positive Stabilization of Linear Differential Algebraic Equation System
}

\author{
Muhafzan \\ Department of Mathematics, Faculty of Mathematics and Natural Sciences, Universitas Andalas, \\ Kampus Unand Limau Manis, Padang 25163, Indonesia
}

Correspondence should be addressed to Muhafzan; muhafzan@fmipa.unand.ac.id

Received 28 April 2016; Accepted 17 July 2016

Academic Editor: Patricia J. Y. Wong

Copyright (C) 2016 Muhafzan. This is an open access article distributed under the Creative Commons Attribution License, which permits unrestricted use, distribution, and reproduction in any medium, provided the original work is properly cited.

We study in this paper the existence of a feedback for linear differential algebraic equation system such that the closed-loop system is positive and stable. A necessary and sufficient condition for such existence has been established. This result can be used to detect the existence of a state feedback law that makes the linear differential algebraic equation system in closed loop positive and stable.

\section{Introduction}

We consider the following linear time invariant differential algebraic equation system:

$$
\begin{aligned}
\frac{d}{d t}(E \mathbf{w}(t)) & =F \mathbf{w}(t)+G \mathbf{u}(t), \\
\mathbf{w}(0) & =\mathbf{w}_{0} \in \mathbb{R}^{n},
\end{aligned}
$$

where $E, F \in \mathbb{R}^{n \times n}$ and $G \in \mathbb{R}^{n \times m}$ are real constant coefficient matrices. In the mathematical control literatures, the variables in (1), namely, $\mathbf{w}(t) \in \mathbb{R}^{n}$ and $\mathbf{u}(t) \in \mathbb{R}^{m}$, denote state and control, respectively. The model of this kind often arises naturally in broad applications because its form is more general than the representations currently used for design and analysis of control systems, so that it is more suitable to describe physical systems. The differential algebraic models are often used in several areas of application such as biology [1] and electrical circuit [2].

As a model, many variables in these systems involve quantities that are intrinsically nonnegative, such as absolute temperatures, concentration of substances in chemical processes, level of liquids in tanks, and number of proteins. These examples belong to the important class of systems which have the property that the state is nonnegative whenever the initial conditions are nonnegative. Hence, the mathematical model describing these systems must take into account this nonnegativity constraint. This leads to the notion of positive differential algebraic equation system [3]. Although positive systems have been actively researched and many results have been reported (see, e.g., [3, 4] and references therein), the literature of positive differential algebraic systems is much more limited. In particular, the fundamental issue of characterizing the stability of positive differential algebraic equation system has only been addressed in [3, 5, 6]. In [3], the stability of positive differential algebraic equation system was investigated by making unnecessary assumptions and general necessary and sufficient conditions were proposed by means of Linear Programming (LP). In [5], the characteristic of feedback of positive LTI continuous singular systems is given only for the case of Index 1 . However, the crucial issue of stabilization still remains unexplored to date. The aim of this paper is to present the first attempt to tackle this important problem. Thus, we present in this short paper a novel approach to detect the existence of a state feedback law that makes the linear differential algebraic equation system in closed loop positive and stable. The remainder of the paper is structured as follows. Section 2 gives formulation of the problem and some basic material required to develop the proposed approach. The main result is considered in Section 3 and finally some conclusions are given. 


\section{Materials and Problem Formulation}

In this section, we present some basic materials which will be needed in the sequel. Shortly, system (1) will be written as a matrix pair $(E, F)$. In the case of $\operatorname{rank}(E)<n$, system (1) is called regular if $\operatorname{det}(\lambda E-F) \neq 0$ for some $\lambda \in \mathbb{C}$, and it is called irregular otherwise. Furthermore, the index of system (1), denoted by ind $(E, F)$, is a smallest nonnegative integer $q$ such that $\operatorname{rank}\left(E^{q+1}\right)=\operatorname{rank}\left(E^{q}\right)$. If the pair $(E, F)$ is regular, then there exists a solution of (1) which is given in [6] as follows:

$$
\begin{aligned}
\mathbf{x}(t)= & \exp \left(\widehat{E}^{D} \widehat{F} t\right) \widehat{E}^{D} \widehat{E} \mathbf{v} \\
& +\int_{0}^{t} \exp \left(\widehat{E}^{D} \widehat{F}(t-\tau)\right) \widehat{E}^{D} \widehat{G} \mathbf{u}(\tau) d \tau \\
& -\left(I-\widehat{E}^{D} \widehat{E}\right) \sum_{i=0}^{q-1}\left(\widehat{E} \widehat{F}^{D}\right)^{i} \widehat{F}^{D} \widehat{G} \mathbf{u}^{(i)}(t),
\end{aligned}
$$

where $\widehat{E}=(\lambda E-F)^{-1} E, \widehat{F}=(\lambda E-F)^{-1} F, \widehat{G}=(\lambda E-F)^{-1} G$, $\mathbf{v} \in \operatorname{im}\left(\widehat{E}^{D} \widehat{E}\right)$, and superscript $D$ denotes the Drazin inverse of corresponding square matrix. Further details on the Drazin inverse can be found in [7].

The differential algebraic equation system (1) with $\operatorname{ind}(E, F)=q$ is called positive if for any admissible initial state $\mathbf{w}_{0} \in \mathbb{R}_{\geq 0}^{n}$ and all control $\mathbf{u}(t) \in \mathbb{R}_{\geq 0}^{m}$ such that $\left(d^{j} / d t^{j}\right)$ $(\mathbf{u}(t)) \in \mathbb{R}_{\geq 0}^{m^{2}}$ with $t \geq 0 ; j=1,2, \ldots, q-1$, then, $\mathbf{w}(t) \in$ $\mathbb{R}_{\geq 0}^{n}$. In addition, it is called stable if $\lim _{t \rightarrow \infty} \mathbf{w}(t)=\mathbf{0}$ for all admissible $\mathbf{w}_{0} \in \mathbb{R}_{\geq 0}^{n}$ [6], where $\mathbb{R}_{\geq 0}^{n}$ denote the set of real vectors of $n$ components in which each component is nonnegative.

In this paper, the problem to deal with is to find a feedback $\mathbf{u}(t)=H \mathbf{w}(t)$ for some matrices $H \in \mathbb{R}^{m \times n}$ for system (1) such that the resulting closed-loop system

$$
\frac{d}{d t}(E \mathbf{w}(t))=(F+G H) \mathbf{w}(t), \quad \mathbf{w}_{0} \in \mathbb{R}^{n}
$$

is regular, positive, and stable.

By referring to $[3,6]$, let us recall the basic background of this type of equation system.

Theorem 1. If $\widehat{E}^{D} \widehat{E} \in \mathbb{R}_{\geq 0}^{n \times n}$, then system (3) with $\mathbf{u}(t)=\mathbf{0}$ is positive if and only if there exists $\alpha \geq 0$ such that

$$
\widehat{E}^{D} \widehat{F}+\alpha \widehat{E}^{D} \widehat{E} \in \mathbb{R}_{\geq 0}^{n \times n}
$$

Theorem 2. If system (3) with $\mathbf{u}(t)=\mathbf{0}$ is positive, then it is stable if and only if there exists $\boldsymbol{\beta} \in \mathbb{R}_{>0}^{n}$ such that $\widehat{E}^{D} \widehat{F} \boldsymbol{\beta} \in \mathbb{R}_{<0}^{n}$.

\section{Results}

In light of Theorems 1 and 2 proposed in $[3,6]$, we have to find a matrix $H \in \mathbb{R}^{m \times n}$ such that

(a) $(\lambda E-\mathscr{F})^{-1}$ exists for some $\lambda \in \mathbb{C}$,

(b) $\bar{E}^{D \overline{\mathscr{F}}}+\alpha \bar{E}^{D} \bar{E} \in \mathbb{R}_{\geq 0}^{n \times n}$ for some $\alpha \geq 0$, (c) $\bar{E}^{D} \overline{\mathscr{F}} \mathbf{x} \in \mathbb{R}_{<0}^{n}$, for some $\mathbf{x} \in \mathbb{R}_{>0}^{n}$, where

$$
\begin{aligned}
& \mathscr{F}=F+G H, \\
& \overline{\mathscr{F}}=(\lambda E-\mathscr{F})^{-1} \mathscr{F}, \\
& \bar{E}=(\lambda E-\mathscr{F})^{-1} E .
\end{aligned}
$$

The existence of these matrices $H \in \mathbb{R}^{m \times n}$ are given in the subsequent result.

Theorem 3. Consider system (1) with (E, F) regular. Then, the following statements are equivalent:

(1) There exists a stabilizing matrix $H \in \mathbb{R}^{m \times n}$ such that the feedback control $\mathbf{u}(t)=H \mathbf{w}(t)$ for system (1) makes the closed-loop system positive and stable.

(2) There exists $\lambda \in \mathbb{C}, \mathbf{x} \in \mathbb{R}_{>0}^{n}$, and $\mathbf{y}_{1}, \mathbf{y}_{2}, \ldots, \mathbf{y}_{n} \in \mathbb{R}^{m}$ such that

(i) $(\lambda E-F-G H)^{-1}$ exists,

(ii) for $i, j=1,2, \ldots, n$, the two things subsequently hold:

$$
\begin{array}{r}
\bar{E}^{D} \bar{F} \mathbf{x}+\bar{E}^{D} \bar{G} \sum_{i=1}^{n} \mathbf{y}_{i} \in \mathbb{R}_{<0}^{n}, \\
\left(\bar{E}^{D} \bar{F}+\alpha \bar{E}^{D} \bar{E}\right)_{(i, j)} x_{i}+\overline{\mathbf{g}}_{i} \mathbf{y}_{i} \in \mathbb{R}_{\geq 0}^{n \times n},
\end{array}
$$

where

$$
\begin{aligned}
& \bar{F}=(\lambda E-F-G H)^{-1} F, \\
& \bar{G}=(\lambda E-F-G H)^{-1} G
\end{aligned}
$$

and $\overline{\mathbf{g}}_{i}$ are the rows of the matrix $\bar{E}^{D} \bar{G}$.

Proof. It is clear that the closed-loop system is given by

$$
\frac{d}{d t}(E \mathbf{w}(t))=(F+G H) \mathbf{w}(t), \quad \mathbf{w}_{0} \in \mathbb{R}^{n}
$$

and $(\lambda E-F-G H)^{-1}$ exists for some $\lambda \in \mathbb{C}$ due to the regularity of $(E, F)$. Note that $\overline{\mathscr{F}}=\bar{F}+\bar{G} H$. Now, define

$$
H=\left[h_{i}\right]_{i=1}^{n}=\left(\begin{array}{llll}
\frac{\mathbf{y}_{1}}{x_{1}} & \frac{\mathbf{y}_{2}}{x_{2}} & \cdots & \frac{\mathbf{y}_{n}}{x_{n}}
\end{array}\right) \in \mathbb{R}^{m \times n} .
$$

From (7), we have

$$
\begin{aligned}
& \left(\bar{E}^{D} \bar{F}+\alpha \bar{E}^{D} \bar{E}\right)_{(i, j)} x_{i}+\overline{\mathbf{g}}_{i} \mathbf{y}_{i} \\
& =\left(\bar{E}^{D} \bar{F}+\alpha \bar{E}^{D} \bar{E}\right)_{(i, j)}+\overline{\mathbf{g}}_{i} \frac{\mathbf{y}_{i}}{x_{i}} \\
& \quad=\left(\bar{E}^{D} \bar{F}+\alpha \bar{E}^{D} \bar{E}\right)_{(i, j)}+\overline{\mathbf{g}}_{i} h_{i} \\
& =\left(\bar{E}^{D} \bar{F}+\alpha \bar{E}^{D} \bar{E}\right)+\bar{E}^{D} \bar{G} H \\
& \quad=\bar{E}^{D}(\bar{F}+\bar{G} H)+\alpha \bar{E}^{D} \bar{E}=\bar{E}^{D} \overline{\mathscr{F}}+\alpha \bar{E}^{D} \bar{E} \in \mathbb{R}_{\geq 0}^{n \times n}
\end{aligned}
$$


which amounts to saying that (7) is equivalent to the positivity of the closed-loop system, that is, condition (b). Furthermore, from (6), we have

$$
\begin{aligned}
\bar{E}^{D} \bar{F} \mathbf{x}+\bar{E}^{D} \bar{G} \sum_{i=1}^{n} \mathbf{y}_{i} & =\left(\bar{E}^{D} \bar{F}+\bar{E}^{D} \bar{G} \sum_{i=1}^{n} \mathbf{y}_{i}(\check{\mathbf{x}})\right) \mathbf{x} \\
& =\left(\bar{E}^{D} \bar{F}+\bar{E}^{D} \bar{G} H\right) \mathbf{x} \\
& =\bar{E}^{D}(\bar{F}+\bar{G} H) \mathbf{x}=\bar{E}^{D} \overline{\mathscr{F}} \mathbf{x},
\end{aligned}
$$

where $\check{\mathbf{x}}=\operatorname{diag}\left(1 / x_{i}\right)$ and $H \mathbf{x}=\sum_{i=1}^{n} \mathbf{y}_{i}$, which amounts to saying that (6) is equivalent to the stability of the closed-loop system, that is, condition (c). rem 3.

Furthermore, let us discuss an example illustrating Theo-

Example 4. Consider the linear differential algebraic equation system in (1) with

$$
\begin{aligned}
& E=\left(\begin{array}{lll}
0 & 0 & 0 \\
0 & 1 & 0 \\
0 & 0 & 1
\end{array}\right), \\
& F=\left(\begin{array}{lll}
1 & 0 & 0 \\
0 & 1 & 0 \\
0 & 0 & -1
\end{array}\right), \\
& G=\left(\begin{array}{l}
0 \\
2 \\
0
\end{array}\right) .
\end{aligned}
$$

It is easy to show that the system under consideration is positive but unstable. Our goal is to design a state feedback controller such that the closed-loop system is positive and stable simultaneously. For this goal, an appropriate matrix $H=\left(\begin{array}{lll}h_{1} & h_{2} & h_{3}\end{array}\right)$ can be obtained from Theorem 3. By a little calculation, we find, for example, $H=\left(\begin{array}{lll}0 & -1 & 0\end{array}\right)$, such that

$$
\begin{aligned}
E & =\left(\begin{array}{lll}
0 & 0 & 0 \\
0 & 1 & 0 \\
0 & 0 & 1
\end{array}\right), \\
F+G H & =\left(\begin{array}{ccc}
1 & 0 & 0 \\
0 & -1 & 0 \\
0 & 0 & -1
\end{array}\right),
\end{aligned}
$$

which is clearly a positive and stable system.

\section{Conclusion}

A necessary and sufficient condition for existence of a feedback for linear differential algebraic equation system such that the closed-loop system is positive and stable has been established. This result can be used to detect the existence of a state feedback law that makes the linear differential algebraic equation system in closed loop positive and stable.

\section{Competing Interests}

The author declares that there is no conflict of interests regarding the publication of this paper.

\section{References}

[1] S. Iwami, Y. Takeuchi, and X. Liu, "Avian-human influenza epidemic model," Mathematical Biosciences, vol. 207, no. 1, pp. $1-25,2007$.

[2] T. R. Ricciardi, D. Salles, W. Freitas, and X. Wang, "Dynamic modeling of inverter-based distributed generators with voltage positive feedback anti-islanding protection," in Proceedings of the iREP Symposium Bulk Power System Dynamics and Control (iREP '10), pp. 1-7, Rio de Janeiro, Brazil, August 2010.

[3] M. Ait Rami and D. Napp, "Characterization and stability of autonomous positive descriptor systems," IEEE Transactions on Automatic Control, vol. 57, no. 10, pp. 2668-2673, 2012.

[4] T. Kaczorek, "Strong stability of positive and compartmental linear systems," Bulletin of the Polish Academy of Sciences, vol. 56, no. 1, pp. 3-7, 2008.

[5] Muhafzan, "On the characterization feedback of positive LTI continuous singular systems of index 1," Advanced Studies in Theoretical Physics, vol. 8, no. 27, pp. 1185-1190, 2014.

[6] E. Virnik, "Stability analysis of positive descriptor systems," Linear Algebra and its Applications, vol. 429, no. 10, pp. 2640$2659,2008$.

[7] S. L. Campbell, C. D. Meyer, and N. J. Rose, "Applications of the Drazin inverse to linear systems of differential equations with singular constant coefficients," SIAM Journal on Applied Mathematics, vol. 31, no. 3, pp. 411-425, 1976. 


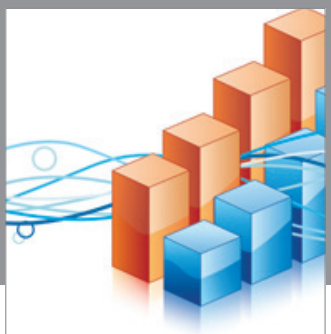

Advances in

Operations Research

vatem alat4

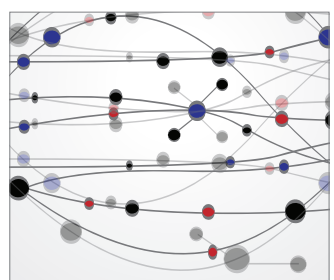

\section{The Scientific} World Journal
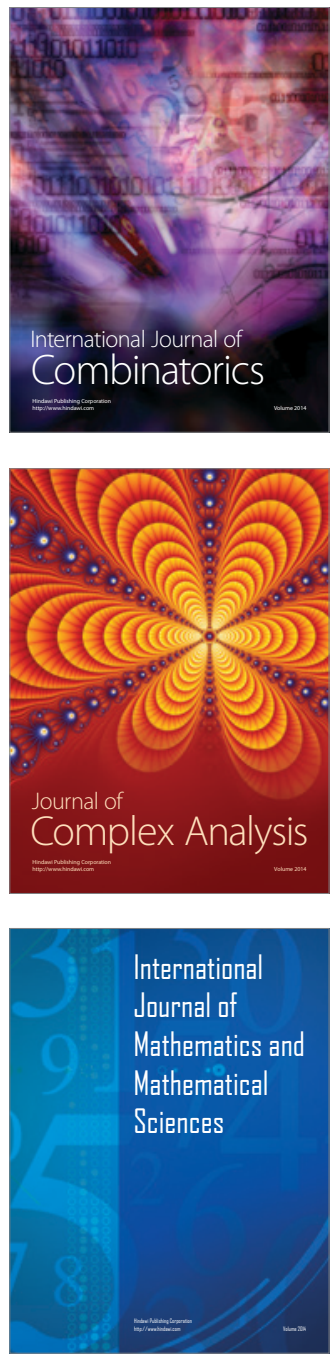
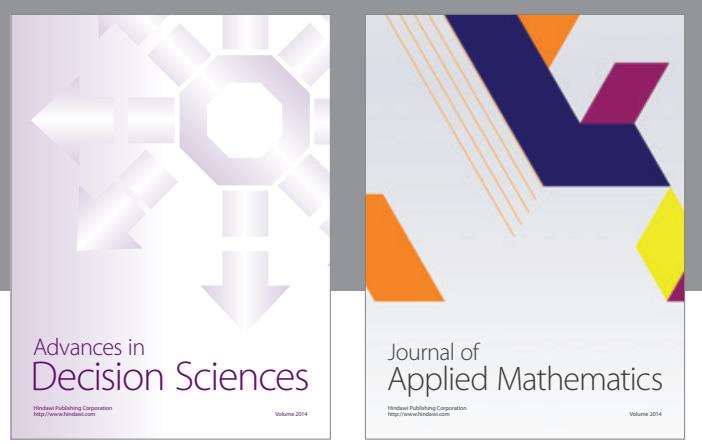

Algebra

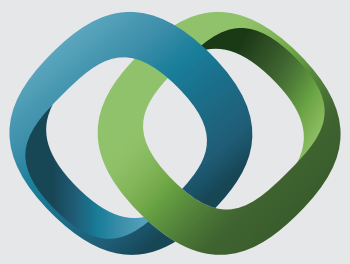

\section{Hindawi}

Submit your manuscripts at

http://www.hindawi.com
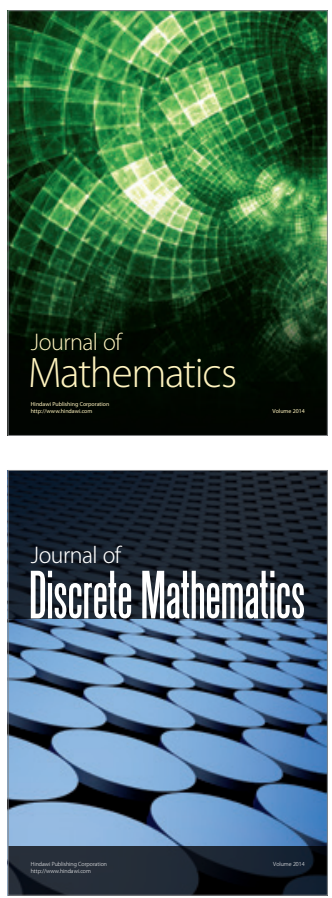

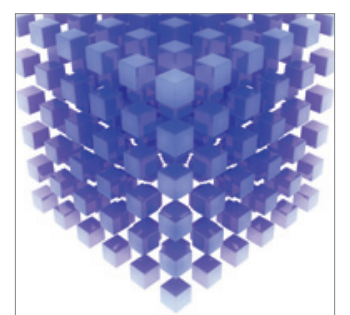

Mathematical Problems in Engineering
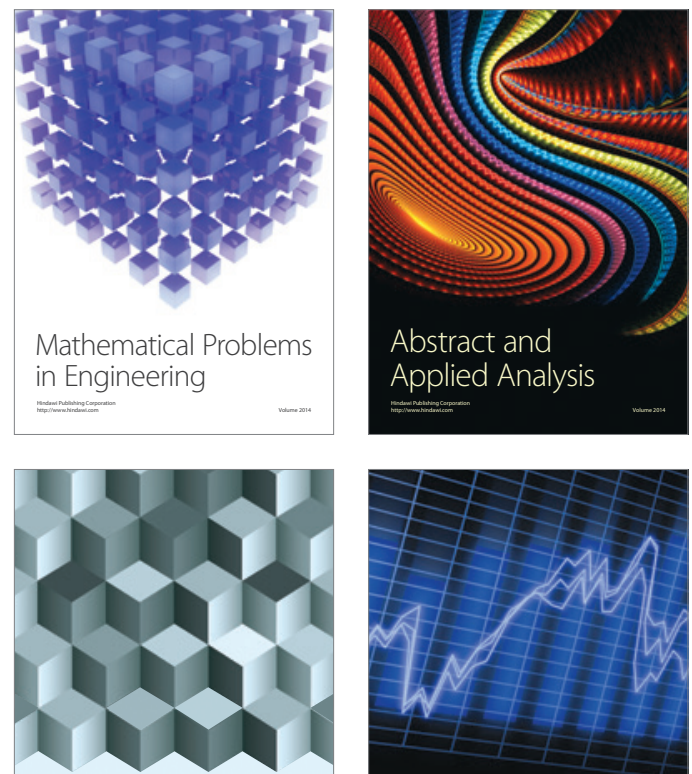

Journal of

Function Spaces

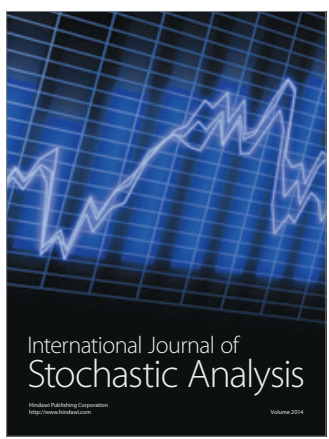

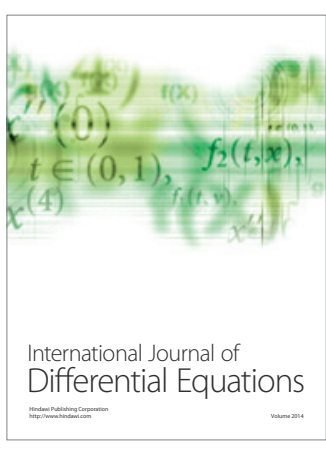
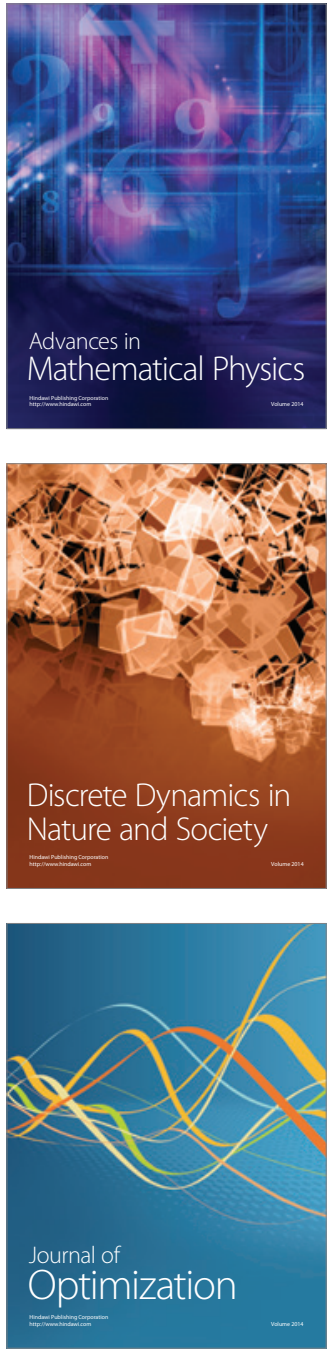